\title{
Phase transition in a chain of quantum vortices
}

\author{
C. Bruder* \\ Institut für Theoretische Festkörperphysik, Universität Karlsruhe, D-76128 Karlsruhe, Germany \\ L. I. Glazman \\ Theoretical Physics Institute, University of Minnesota, Minneapolis, Minnesota 55455 \\ and Delft University of Technology, 2600 GA Delft, The Netherlands \\ A. I. Larkin \\ Theoretical Physics Institute, University of Minnesota, Minneapolis, Minnesota 55455 \\ and L. D. Landau Institute for Theoretical Physics, Moscow 117940, Russia \\ J. E. Mooij and A. van Oudenaarden \\ Delft University of Technology, 2600 GA Delft, The Netherlands
}

(Received 8 September 1998)

\begin{abstract}
We consider interacting vortices in a quasi-one-dimensional array of Josephson junctions with small capacitance. If the charging energy of a junction is of the order of the Josephson energy, the fluctuations of the superconducting order parameter in the system are considerable, and the vortices behave as quantum particles. Their density may be tuned by an external magnetic field, and therefore one can control the commensurability of the one-dimensional vortex lattice with the lattice of Josephson junctions. We show that the interplay between the quantum nature of a vortex and the long-range interaction between the vortices leads to the existence of a specific commensurate-incommensurate transition in a one-dimensional vortex lattice. In the commensurate phase an elementary excitation is a soliton with energy separated from the ground state by a finite gap. This gap vanishes in the incommensurate phase. Each soliton carries a fraction of a flux quantum; the propagation of solitons leads to a finite resistance of the array. We find the dependence of the resistance activation energy on the magnetic field and parameters of the Josephson array. This energy consists of the above-mentioned gap, and also of a boundary pinning term, which is different in the commensurate and incommensurate phases. The developed theory allows us to explain quantitatively the available experimental data. [S0163-1829(99)00402-6]
\end{abstract}

\section{INTRODUCTION}

The interest in Josephson junction arrays in the last decade was to a large degree prompted by the fact that these systems are suitable as a testing ground for various predictions of quantum many-body theory (for an overview see, e.g., Refs. 1 and 2). If the charging energy of a junction $E_{C}=e^{2} / 2 C$ is comparable with its Josephson energy $E_{J}$, the phase of the superconducting order parameter is subject to quantum fluctuations (here $C$ is the capacitance of a junction). At some critical value of $E_{C} / E_{J}$ the global phase coherence is destroyed, and the array becomes an insulator. ${ }^{3}$ This transition apparently is driven by proliferation of spontaneously created vortices, i.e., topological excitations of the array, in which the phase of the order parameter varies by $2 \pi$ on going around a plaquette. At smaller ratios $E_{C} / E_{J}$ vortices induced by an external magnetic field still possess quantum properties. The vortex dynamics is particularly sensitive to the quantum fluctuations of the phase: the vortex mass, for example, is finite entirely due to these fluctuations. ${ }^{4,5}$

A single vortex in a Josephson junction array behaves as a ballistically propagating quantum particle. ${ }^{6}$ These particles are strongly interacting, however: for the values of $E_{C} / E_{J}$ $\lesssim 1$ at which the global phase coherence is preserved, their interaction energy $U_{v-v} \propto E_{J}$ is larger than the bandwidth for a single vortex. A finite magnetic field applied perpendicularly to the array, creates a lattice with a vortex density proportional to the field strength. Depending on the magnetic field flux per plaquette, the vortex lattice is commensurate or incommensurate with the junction array. ${ }^{7}$ The commensurability effect exists of course even for classical vortices in an array with $E_{C} / E_{J} \rightarrow 0$. The array acts like a periodic potential with an amplitude $U_{p} \sim 0.2 E_{J}$ and some period $a$ (the period of the Josephson array) for each vortex. ${ }^{8}$ In a classical system, this is expected to be a source of strong pinning, as $U_{p}$ and $U_{v-v}$ are of the same order. Quantum fluctuations bring new physics into the problem. The period of the pinning potential is relatively small, and therefore its amplitude is suppressed readily by quantum fluctuations. On the contrary, depending on the magnetic field, the vortex lattice period may be significantly larger than $a$, thus making the vortex lattice robust against quantum fluctuations.

Commensurability effects in a chain of quantum vortices were investigated in the recent experiments of van Oudenaarden et al. ${ }^{9}$ There a number of two-dimensional arrays with various ratios $E_{C} / E_{J}$, and various widths of the order of ten cells were studied. All arrays were quasi-one- 


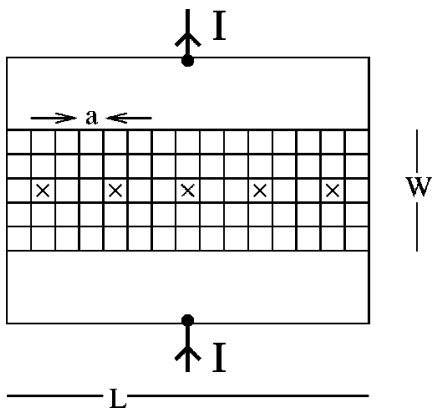

FIG. 1. Quasi-one-dimensional Josephson array. Each side of a plaquette corresponds to a single Josephson junction. Crosses denote vortices located in the central row of the array. The properties of the vortex chain are probed by passing a current $I$ from one superconducting contact to the other and measuring the voltage between them. The array width is $W$ and the size of a single plaquette is $a \times a$.

dimensional in fact, their length varying between 100 and 1000 cells. Superconducting contacts parallel to the long sides of the array were providing a potential confining the vortices to the central row of the array; see Fig. 1. Applying a current through the leads (perpendicular to the onedimensional vortex chain) and measuring the resistance of the system as a function of the magnetic field, van Oudenaarden et al. found almost zero resistance in the regions centered around the commensurate values of the onedimensional vortex density. This was interpreted as an indication of a finite-gap state ("Mott insulator" phase), induced by the vortex-vortex interaction in the presence of a periodic potential. If the magnetic field was tuned away from these special regions, a transition to a resistive state was observed, indicating moving vortices ("conducting" phase).

The analogy between the observed transition in the system of vortices and the textbook Mott transition in electron systems can be made more explicit: $E_{C} / E_{J}$ here plays the role of the ratio $t / U$ of the electron bandwidth to the on-site repulsion potential; the magnetic field $B$, creating vortices, plays the role of the electron chemical potential $\mu$. The phase diagram in the variables $(t / U, \mu)$ consists of two phases. The insulating phase occurs at relatively small values of $t / U \lesssim 1$. In this phase, the electron density is constant as a function of $\mu$, and fixed by the commensurability condition (one electron per lattice site). One can assign the value $\mu$ $=0$ to the line of particle-hole symmetry in this phase diagram. Deviation from this symmetry line makes the excitation gap in the insulating phase smaller; the gap width is a nonanalytical function of $\mu$, reflecting the violation of the particle-hole symmetry at $\mu \neq 0$. At a certain critical value of $|\mu|$, which depends on $t / U$, the gap disappears and a transition to a "conducting" phase occurs. This conventional picture is modified somewhat in the one-dimensional case, but qualitatively remains valid. A similar description applies to Mott transitions in Bose systems with repulsion. ${ }^{10}$

The line of particle-hole symmetry in the case of vortices corresponds to a special value $B_{0}$ of the magnetic field, which induces a vortex lattice commensurate with the period of the junction array. The analogy to the electronic case described in the last paragraph suggests that the vortex density remains constant in a finite interval of $B$ around $B_{0}$. One also expects a gap in the excitation spectrum of the array that diminishes as a function of $\left|B-B_{0}\right|$ within the commensurate phase (which is the analog of the insulating phase). Indeed, in the experiment a cusplike dependence of the resistance activation energy on $\left|B-B_{0}\right|$ was observed (see Ref. 9 and Sec. VI of this paper). At some critical value of $\mid B$ $-B_{0} \mid$ the observed temperature dependence of the resistance becomes considerably weaker. This may indicate that a transition to a gapless ("conducting") phase occurs, accompanied by the creation of discommensuration solitons.

In this paper, we present a quantitative theory of the commensurate-incommensurate transition for a chain of quantum vortices in a quasi-one-dimensional Josephson array (see Ref. 11 for another type of commensurateincommensurate transition in Josephson arrays). The transition to the incommensurate state occurs by proliferation of the discommensuration solitons through the vortex chain. We are able to develop a theory by analytical means because of a special feature of the system we consider. It turns out that the range of the interaction between the vortices is much longer than the intervortex distance. Therefore, the solitons consist of many vortices, and possess a large effective mass. Thus the theory for the commensurate-incommensurate transition is essentially classical. However, to relate the parameters of this theory to the generic properties $\left(E_{C}\right.$ and $\left.E_{J}\right)$ of the Josephson array, we need to consider a single vortex as a quantum particle: the amplitude of the periodic pinning potential depends on the bandwidth of the vortex. After that, we are able to find analytically the excitation gap existing in the commensurate phase and the boundaries of this phase in the $\left(B, E_{C} / E_{J}\right)$ plane.

We relate the characteristics of the commensurate and incommensurate phases to an observable quantity, the activation energy of the resistance $E_{R}$. In the commensurate phase, the transfer of one flux quantum between the edges of the array occurs via a sequence of solitons propagating through it. The number of solitons necessary to transfer one vortex is equal to the ratio of the periods of the vortex lattice and the junction array; typically this ratio is large. At any time during the vortex transfer, there is no more than one soliton present in the chain. We demonstrate that $E_{R}$ depends not only on the properties of the "bulk" one-dimensional system, but also reflects boundary pinning effects, accompanying the passage of vortices through the ends of the array. One soliton changes the length of the vortex chain only by one period of the junction array, which is less than the intervortex spacing. Hence, in the commensurate phase, the process of vortex flow through the array can be viewed as motion of a rigid vortex chain. Because of the rigidity, the vortex chain cannot adjust itself to the boundary pinning potential. The potentials produced by the two ends of the array add to $E_{R}$ : the relative phase of these two contributions depends on whether the total flux piercing the junction array equals an integer number of flux quanta. Thus, in the commensurate state, there are two major terms in $E_{R}$. The first term is the activation energy of a soliton, and the second term is the sum of the boundary pinning energies. This second (smaller) term oscillates with the magnetic flux piercing the array. In the incommensurate state, the vortex chain is compressible, and can adjust to the boundaries of the array, if the latter is sufficiently long. As a result, the main term in $E_{R}$ is the bound- 
ary pinning potential, which a vortex has to overcome to enter the array. A correction is provided by the finite compression energy of the chain. Its average value depends on the compressibility of the chain, renormalized by the solitons, and is inversely proportional to the length of the array. This term oscillates with the flux threading the system.

The paper is organized as follows. In Sec. II, we introduce a model of classical vortices in the quasi-one-dimensional Josephson array. Here we neglect the discreteness of the array, and the screening of the vortex-vortex interaction. This approximation means that the vortex chain is entirely incompressible. We establish the stability criterion for a onedimensional vortex chain against formation of a zigzag structure. We calculate the equilibrium number of vortices as a function of the magnetic field and determine the boundary pinning caused by the interaction of the vortices with the ends of the array. For an incompressible chain, this gives us the equilibrium position for each vortex. In the following part, Sec. III, we discuss bulk pinning by reintroducing the discreteness of the junction array. The array creates a periodic potential for each vortex, which behaves as a quantum particle in this potential. We demonstrate that typically the amplitude of the quantum fluctuations of a vortex exceeds the period of the array. (This justifies, in fact, the approximations made in Sec. II.) We calculate the residual pinning potential, suppressed by quantum and thermal fluctuations, acting on a single vortex.

The results of the Secs. II and III are directly applicable to short arrays, i.e., arrays that are shorter than the range of the vortex-vortex interaction. The main goal of these sections though is to provide us with the coefficients necessary to write down the effective Hamiltonian describing a compressible chain in a long array. We start the next section, Sec. IV, with an estimate of the vortex-vortex interaction range. It is defined by two mechanisms: (1) the effect of the magnetic field induced by the vortices, and (2) the interaction of the vortices in the Josephson array with the Abrikosov lattice in the contacts to the array. The estimate demonstrates that for the conditions of the experiments ${ }^{9}$ the range indeed exceeds greatly the intervortex distance, but still may be smaller than the system length, making it necessary to account for a finite compressibility of the vortex chain. We therefore derive the long-wavelength theory for the compressible vortex chain. This theory enables us to describe, in Sec. V, the commensurate-incommensurate transition. We determine the boundaries of the commensurate phase, and find the dependence of the activation energy for elementary excitations on the parameters of the system. Also in this section, we discuss the behavior of the resistivity following from the picture we developed. We compare our results with the existing experiment in Sec. VI. Using the experimental values of the vortex density at the commensurate-incommensurate transition and the maximum of the activation energy of the resistance, we are able to give parameter-free estimates of the range of the vortex-vortex interaction and of the elastic constant of the vortex chain. The effective pinning potential turns out to be at least an order of magnitude smaller than the bare potential due to quantum fluctuations (as calculated in Sec. III). The soliton length is extremely large and of the order of the length of the array. The long-range nature of the vortexvortex interaction leads to a large value of the elastic con- stant: the chain is virtually rigid in the incommensurate phase. Our theory explains consistently the main experimental observations reported in this paper and in Ref. 9: (1) the cusplike dependence of the activation energy on the magnetic field in the commensurate phase; (2) the large value of this activation energy (compared to $E_{J}$ and $E_{C}$ ), and (3) oscillations of the resistance with the applied magnetic field, with a period corresponding to one flux quantum through the entire array, in the incommensurate phase. We conclude with a discussion in Sec. VII.

\section{RIGID VORTEX CHAIN}

We consider a two-dimensional Josephson array of lattice constant $a$, length $L$, and width $W$ where $L \gg W$; see Fig. 1 . The "sites" of this array are superconducting islands, linked by Josephson junctions that are characterized by a capacitance $C$ and a critical current $I_{C}$. The phases $\varphi_{\mathbf{i}}$ of the order parameter of the islands (numbered by vectors $\mathbf{i}$ ) are the only dynamical degrees of freedom of the system. For an infinite two-dimensional system, the Lagrangian can be written in the standard ${ }^{4}$ way,

$$
L=\sum_{\langle\mathbf{i}, \mathbf{j}\rangle}\left\{\frac{\hbar^{2}}{8 E_{C}}\left(\frac{\partial \varphi_{\mathbf{i}, \mathbf{j}}}{\partial t}\right)^{2}-E_{J}\left[1-\cos \left(\varphi_{\mathbf{i}, \mathbf{j}}\right)\right]\right\} .
$$

Here the sum is taken over the nearest neighbors, and $\varphi_{\mathbf{i}, \mathbf{j}}$ is the phase difference across a link of the array, $E_{J}$ $=I_{C} \Phi_{0} / 2 \pi$ and $E_{C}=e^{2} / 2 C$ are the Josephson and charging energy, respectively; $\Phi_{0}=h c / 2 e$ is the flux quantum. The Lagrangian (1) describes quantum fluctuations of the phase in the array. At a certain critical value ${ }^{12}$ of the ratio $E_{C} / E_{J}$ $\sim 1$, the proliferation of spontaneous vortices and antivortices through the system destroys the long-range order. We consider smaller values of $E_{C} / E_{J}$, and neglect the existence of spontaneous topological excitations. Vortices in the spatial distribution of the phase $\varphi$ are then induced only by an external magnetic field $B$. A vortex is characterized by a phase change of $2 \pi$ on going around a plaquette. The effective Lagrangian in terms of the vortex positions,

$$
L=\sum_{i} \frac{M}{2}\left(\frac{d \mathbf{r}_{i}}{d t}\right)^{2}-\sum_{i, j} \frac{1}{2} U_{v-v}\left(\mathbf{r}_{i}, \mathbf{r}_{j}\right)-\sum_{i} U_{p}\left(\mathbf{r}_{i}\right)
$$

can be derived ${ }^{4}$ from Eq. (1). Here $M=\pi^{2} \hbar^{2} / 4 a^{2} E_{C}$ is the vortex mass, $U_{v-v}\left(\mathbf{r}_{i}, \mathbf{r}_{j}\right)$ is the interaction energy between the vortices, and $U_{p}\left(\mathbf{r}_{i}\right)$ is the pinning potential that represents the effect of a discrete lattice of junctions on the vortex motion. In an infinite array, the energy $U_{v-v}$ depends only on the distance between vortices, and can be approximated by the standard expressions valid for vortices induced in a thin superconducting film. ${ }^{13}$ For a geometrically restricted array, Fig. 1, the form of the interaction potential $U_{v-v}$ depends crucially on the boundary conditions for the phase that are set by the massive superconducting contacts. The superfluid density in these superconducting strips exceeds greatly the effective superfluid density in the array. Therefore, each vortex in the array is repelled from the boundaries [this is represented by the terms $U_{v-v}\left(\mathbf{r}_{i}, \mathbf{r}_{i}\right) \equiv U_{v-v}\left(y_{i}\right)$ in the Lagrangian]. At a sufficiently weak magnetic field, $B \leqq \Phi_{0} / W^{2}$, the intervortex distance is large enough, and vortices occupy only the central row of the array. 
The pinning potential in this one-dimensional case may be modeled $^{8}$ by a function of $x$ only,

$$
U_{p}(x)=0.1 E_{J}[1-\cos (2 \pi x / a)] .
$$

We will see in the next section that quantum fluctuations of the vortex positions strongly diminish the role of pinning by the periodic lattice of Josephson junctions. For now, we will ignore the contribution of the pinning potential, given by the third term in the Lagrangian, Eq. (2).

Because of the large superfluid density in the contacts, the phase of the order parameter varies only slightly along each of the long boundaries of the array. Currents induced by a vortex in the array flow through it almost perpendicularly to the boundaries. In the limit of infinite superfluid density and infinite London-Pearl penetration depth ${ }^{13}$ in the contacts, the currents within the array do not decay with the distance from a vortex. As a result, the range of the vortex-vortex interaction is infinite. The interaction potential, up to an arbitrary constant, has the following form:

$$
U_{v-v}=-2 \pi^{2} E_{J}\left|x_{i}-x_{j}\right| / W
$$

at $\left|x_{i}-x_{j}\right| \gtrsim W$. Because of this form, the vortex chain is absolutely rigid at small wave vectors.

If the smallest intervortex distance exceeds the array width, one can use the limiting form of the potential (4) to calculate the contribution of the vortex-vortex interaction [the second term in Eq. (2)] to the energy of the vortex chain. It is more convenient, however, to write down this energy directly in terms of the phase distribution in the array:

$$
\frac{\partial \varphi}{\partial y}(x)=\frac{1}{W}\left(2 \pi n x-\pi \sum_{i=1}^{N} \operatorname{sgn}\left(x-x_{i}\right)+\varphi_{0}\right) .
$$

Here, we have replaced the phases $\varphi_{\mathbf{i}}$ of the islands by a continuous variable $\varphi(\mathbf{r})$. The form (5) of the phase gradient is valid at distances $\left|x-x_{i}\right| \geqslant W$ away from the vortex centers $x_{i}$. The magnetic field enters via the one-dimensional density $n=B W / \Phi_{0}$. The phase $\varphi_{0}$ has the meaning of the average phase difference between the contacts, and will be used as a Lagrange multiplier to enforce the condition of fixed current $I$ in the $y$ direction through the array. The typical shape of the phase gradient is illustrated in Fig. 2. The energy in the presence of a current $I$ between the contacts can be written as

$$
E\left(\left\{x_{i}\right\}, \varphi_{0}\right)=\frac{E_{J} W}{2} \int_{-L / 2}^{L / 2} d x\left(\frac{\partial \varphi}{\partial y}\right)^{2}-\frac{\Phi_{0}}{2 \pi} I \varphi_{0} .
$$

The equilibrium positions of the vortices and $\varphi_{0}$ for a given value of the current $I$ are defined by the set of conditions

$$
\begin{aligned}
& \frac{\partial E\left(\left\{x_{i}\right\}, \varphi_{0}\right)}{\partial x_{i}}=0, \\
& \frac{\partial E\left(\left\{x_{i}\right\}, \varphi_{0}\right)}{\partial \varphi_{0}}=0 .
\end{aligned}
$$

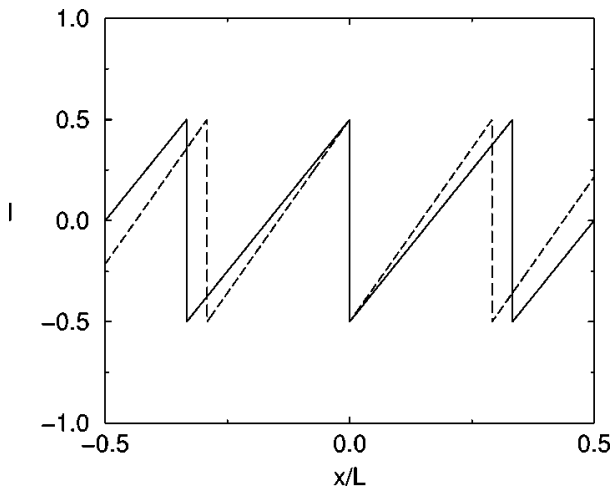

FIG. 2. Current (in arbitrary units) across the array as a function of the coordinate $x$ along the array. Note the jumps at the vortex positions $x_{i}$. For $n L=$ integer (solid line), we can identify the two edges at $\pm L / 2$, and shifting the vortex chain along the $x$ direction does not change the energy, Eq. (6). In contrast to that, for $n L$ $\neq$ integer (dashed line), the energy depends on the position of the chain (boundary pinning).

At first we will consider the case $I=0$ and will determine the equilibrium number $N$ and positions $x_{i}^{0}, i=1, \ldots, N$ of vortices in the array. Solving Eqs. (7), we obtain ${ }^{14}$

$$
N=\mathcal{I}(n L),
$$

where $\mathcal{I}(x)$ is the integer part of $x$. We will consider only positive values of the magnetic field, $n>0$. The equilibrium positions are given by

$$
x_{i}^{0}=\frac{2 i-1-N}{2 n},
$$

which means that the vortices are equidistant, $x_{i+1}^{0}-x_{i}^{0}$ $=1 / n$. The first and the last vortex of the chain are located at a distance $[n L-\mathcal{I}(n L)+1] /(2 n) \geqslant 1 /(2 n)$ away from the ends of the array. On increasing the flux, they move towards the center.

For deviations of the vortex coordinates from their equilibrium positions, the energy Eq. (6) may be expressed as

$$
E=E_{0}+\frac{2 \pi^{2} E_{J} n}{W}\left(\bar{x}^{2} \mathcal{F}(n L)+\sum_{i=1}^{N}\left(x_{i}-x_{i}^{0}\right)^{2}\right),
$$

where $\bar{x}=\sum_{i} x_{i} / N$ is the center of mass of the vortex chain; for brevity, hereafter we use the notation

$$
\mathcal{F}(n L) \equiv n L-\mathcal{I}(n L) .
$$

Each individual vortex resides in a parabolic well, centered at the vortex equilibrium position; this is the result of the infinite-range interaction between the vortices.

The term proportional to $\bar{x}^{2}$ is caused by the interaction of the vortices with the two boundaries at $\pm L / 2$ : if $n L$ is integer, shifting the vortex chain along the $x$ direction does not change the energy, Eq. (6); see Fig. 2. For general values of $n L$, the energy depends on the position of the chain. That means that the boundaries pin the vortex chain.

The activation energy of the system is given by the difference in ground-state energies $E_{0}$ of the $N+1$ and $N$ vortex chains at a given value of the flux density $n$. A straightforward calculation starting with Eq. (6) at $I=0$ yields 


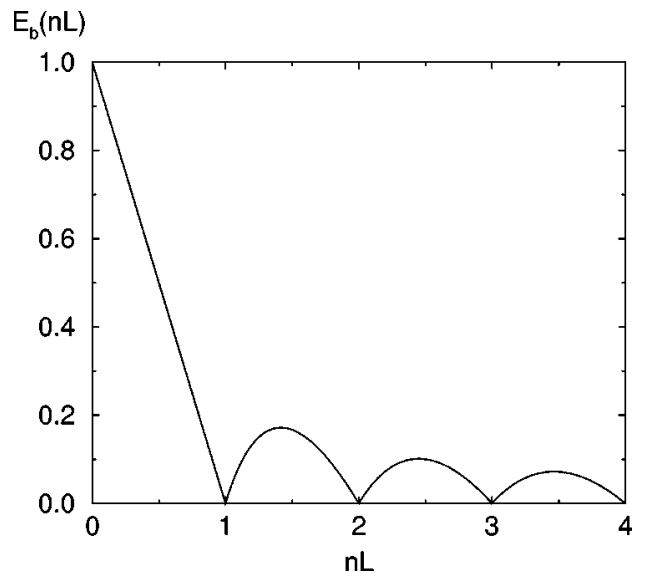

FIG. 3. Activation energy (in units of $\pi^{2} E_{J} L / 2 W$ ) of the rigid chain as a function of $n L=B W L / \Phi_{0}$. The critical current is given by the same curve: $I_{C}^{\text {array }}=4 E_{b}(n L) / \Phi_{0}$; see Eq. (14) and also Ref. 15 .

$$
E_{b}(n L)=\frac{\pi^{2} E_{J}}{2 W n}[1-\mathcal{F}(n L)] \mathcal{F}(n L) .
$$

The boundary pinning energy (12) vanishes for integer values of $n L$; the maxima between two zeros are $\pi^{2} E_{J} /(8 n W)$, and decay with the magnetic field as $1 / n$; see Fig. 3 .

It is also possible to calculate the critical current of the array, $I_{C}^{\text {array }}$. We define it as the current at which the stability of the center of mass $\bar{x}$ is lost. The relation between $I$ and $\bar{x}$ can be found from Eq. (6),

$$
I=\frac{2 \pi E_{J}}{\Phi_{0}} \int_{-L / 2}^{L / 2} d x\left(\frac{\partial \varphi}{\partial y}\right)=\frac{4 \pi^{2} E_{J} \bar{x}}{\Phi_{0} W} \mathcal{F}(n L) .
$$

The center-of-mass stability requires that no vortex is to enter or leave the system; this restriction leads to

$$
I_{C}^{\mathrm{array}}=\frac{4 E_{\mathrm{b}}}{\Phi_{0}} \equiv \frac{\pi I_{C}}{n W}[1-\mathcal{F}(n L)] \mathcal{F}(n L) .
$$

This functional dependence of the critical current on $n L$ for the array coincides with the one obtained in Ref. 15 for a thin-film bridge.

It is clear that the one-dimensional approximation breaks down for large magnetic fields, i.e., if the vortex-vortex distance becomes considerably less than the width $W$ of the array. To obtain a quantitative value of the critical field, we studied the instability of the vortex chain towards formation of a zigzag deformation. For that purpose, we derive the full formula for $U_{v-v}\left(\mathbf{r}_{i}, \mathbf{r}_{j}\right)$, which requires properly taking into account the infinite number of image vortices necessary to fulfill the boundary conditions at the superconducting contacts. After that, we replace Eq. (6) by its two-dimensional analog, which depends on the two-dimensional vectors of displacements of each vortex. An analysis of the dependence of this energy on the transverse vortex displacements yields the value of the critical field at which the zigzag pattern forms:

$$
B_{\text {crit }}=\frac{\Phi_{0}}{0.65 W^{2}} .
$$

In other words, the vortex distance has to be larger than $0.65 \mathrm{~W}$ for the one-dimensional approximation to be valid.

\section{PINNING BY THE PERIODIC POTENTIAL}

In the last section, the discreteness of the system was neglected completely. At first sight, this seems to be an unreasonable approximation. Indeed, only at $n W>$ $\left(0.4 / \pi^{2}\right)(W / a)^{2}$ the amplitude $0.2 E_{J}$ of the pinning potential (3) is smaller than the variation $\delta E$ of the energy (10) if a single vortex is displaced by $a / 2(a / 2$ is the distance between the minimum and maximum of the pinning potential). Note that for the stability of a single-row vortex chain, the condition $n W<1.62$ must be satisfied [see Eq. (15)]. The two restrictions on $n W$ are incompatible except for quite narrow arrays, $W / a \leqslant 6$. In this section, we demonstrate that the effective pinning potential is reduced significantly by quantum fluctuations of the vortex coordinates, which makes the above restriction irrelevant, even at relatively small ratios $E_{C} / E_{J}$.

Forgetting the interactions with the other vortices for a moment, each vortex is described by a Hamiltonian

$$
H=\frac{p_{i}^{2}}{2 M}+0.1 E_{J}\left[1-\cos \left(2 \pi x_{i} / a\right)\right]
$$

That means, it is a delocalized quantum particle characterized by a band structure $\epsilon(k)$. In the limit of small quantum fluctuations of the phase, $E_{C} \lesssim 0.4 E_{J}$, the problem can be treated in the tight-binding approximation. ${ }^{16}$ This leads to the following expression for the dispersion relation:

$$
\epsilon(k)=-\frac{E_{p}}{2} \cos (k a),
$$

where the bandwidth is given by

$$
E_{p}=\frac{8}{\pi} \sqrt{0.1 E_{J} E_{C}} \exp \left(-2 \sqrt{0.1 E_{J} / E_{C}}\right),
$$

and the effective mass of the vortices in the periodic potential by

$$
m_{\mathrm{eff}}^{-1}=\frac{a^{2} E_{p}}{\hbar^{2}} .
$$

At stronger fluctuations the tight-binding approximation is inadequate, and the bandwidth becomes of the order of $E_{C}$. We note that $E_{p}$ can be interpreted as a transition amplitude. The exponent of this amplitude can be also extracted from Ref. 4, where the rate of transitions between two adjacent minima of the pinning potential was estimated. According to Ref. 4, this exponent is approximately $2.25 \sqrt{E_{J} / 8 E_{C}}$, which is about $10 \%$ higher than the exponent in Eq. (18).

In Eq. (10) we showed that each vortex moves in a parabolic potential produced by the interaction with the other vortices. The corresponding oscillation frequency of a particle having effective mass $m_{\text {eff }}$ is

$$
\omega_{\mathrm{osc}}=\sqrt{\frac{4 \pi^{2} E_{J} n}{W m_{\mathrm{eff}}}}=\frac{1}{\hbar} \sqrt{2 \pi^{2} E_{J} E_{p} n a^{2} / W},
$$

and the mean-square oscillation amplitude is 


$$
\left\langle\left(x_{i}-x_{i}^{0}\right)^{2}\right\rangle=\frac{\hbar}{m_{\mathrm{eff}} \omega_{\mathrm{osc}}}=a \sqrt{\frac{E_{p} W}{8 \pi^{2} E_{J} n}} .
$$

The quantum fluctuations implied by Eq. (21) lead to a reduction of the effective pinning potential, which becomes now a (periodic) function of $x_{i}^{0}$. Estimating the effective pinning, we assume that the intervortex interaction on the scale of the lattice constant $a$ is weak compared to $E_{p}$. In accordance with the standard prescription of solid-state physics, ${ }^{16}$ we replace the (quasi) wave vector in the dispersion relation, Eq. (17), by an operator $\hat{p} / \hbar$, and consider the Schrödinger equation for a quasiparticle with Hamiltonian

$$
\widetilde{H}=\epsilon\left(\frac{\hat{p}}{\hbar}\right)+\frac{1}{2} m_{\mathrm{eff}} \omega_{\mathrm{osc}}^{2}\left(\hat{x}-x_{i}^{0}\right)^{2} .
$$

Here $\hat{p}$ and $\hat{x}$ are canonically conjugate variables. But now we can view $\hat{x}$ as the momentum of some particle, moving in the potential $\epsilon(p / \hbar)$ that is periodic in the coordinate $p$ of the particle. Therefore, eigenstates of the Hamiltonian (22) with various values of the "quasimomentum" $x_{i}^{0}$ form bands. For each band, the energy is a periodic function of $x_{i}^{0}$ with period $a$. At zero temperature, we are interested in the lowest band, with the energy

$$
U_{p}^{\mathrm{eff}}\left(x_{i}^{0}\right)=U_{p}^{\mathrm{eff}} \cos \left(2 \pi x_{i}^{0} / a\right) .
$$

The value of $U_{p}^{\text {eff }}$ depends on the magnitude and form of the periodic potential $\epsilon(p / \hbar)$. Using (17) and (18), we find

$$
U_{p}^{\mathrm{eff}}=E_{J} \frac{a}{W} \sqrt{2 n W \frac{E_{p}}{E_{J}}} \exp \left(-\frac{2 \sqrt{2}}{\pi} \frac{W}{a} \sqrt{\frac{E_{p}}{E_{J}} \frac{1}{n W}}\right) .
$$

Equation (23) gives the effective pinning potential for a single vortex. It is worth noting that the pinning strength diminishes with the increase of the equilibrium intervortex distance $1 / n$.

The approximations we employed in deriving the form [Eq. (23)] and amplitude [Eq. (24)] of the pinning potential require a sufficiently wide band for the motion of a vortex in the periodic potential. In other words, the exponential factor in Eq. (24) must be small. In the opposite limit of negligible quantum fluctuations, the magnitude of the effective potential is $0.2 E_{J}$, and the function $U_{p}^{\text {eff }}\left(x_{i}^{0}\right)$ has cusps at $x_{i}^{0}$ coinciding with the maxima of the bare potential $U_{p}(x)$ defined in Eq. (3). Each cusp in $U_{p}^{\text {eff }}\left(x_{i}^{0}\right)$ corresponds to a jump of the coordinate of a classical vortex between the minima of the potential $U_{p}(x)$.

One may get an idea of how effective the quantum smearing is by estimating $U_{p}^{\text {eff }}$ at $W / a=10$ and $E_{C} \simeq 0.4 E_{J}$, which is close to the limit of applicability of Eq. (18). Substitution of these values in Eq. (24) yields

$$
U_{p}^{\mathrm{eff}} \simeq 0.056 \sqrt{n W} E_{J} \exp (-3.58 / \sqrt{n W}) .
$$

In this example, the effective pinning potential gets smaller than its bare value at $1 / n \gtrsim W / 40$, which is always the case in practice.
At finite temperature $T \gtrsim \hbar \omega_{\text {osc }}$, we have to consider the averaging of the periodic potential by quantum and thermal fluctuations; these further reduce the pinning. Summing the geometric series, we obtain

$$
U_{p}^{\mathrm{eff}}(T)=U_{p}^{\mathrm{eff}} \frac{1}{1+\exp \left(-\hbar \omega_{\mathrm{osc}} / T\right)} .
$$

We will now calculate the pinning of the rigid vortex chain. Each vortex is subject to the potential Eq. (23). Summing over the members of the chain [which are located at the positions shifted by $\bar{x}$ from the equilibrium values (9)] leads to

$$
\begin{aligned}
U_{\text {pin }}(\bar{x}) & =\sum_{i=1}^{N} U_{p}^{\mathrm{eff}}\left(x_{i}^{0}+\bar{x}\right) \\
& =-U_{p}^{\mathrm{eff}}(T) \cos (2 \pi \bar{x} / a) \frac{\sin (N \pi / n a)}{\sin (\pi / n a)} .
\end{aligned}
$$

For commensurate values of the flux, i.e., if $1 / n a$ is integer, we get

$$
U_{\text {pin }}(\bar{x})=-U_{p}^{\mathrm{eff}}(T) \cos (2 \pi \bar{x} / a) N ;
$$

the pinning barrier is proportional to the total number of vortices, i.e., the pinning is strong. In the immediate neighborhood of the commensurate points, however, there are values of $n$ for which

$$
n=\frac{\mathcal{I}(n L)}{a} .
$$

At these vortex densities, the numerator of Eq. (26) vanishes, i.e., there is no pinning. The spacing between these zeros is approximately given by $n a / L$, which may be less than $1 / L$. The rapid oscillations are caused by the fact that we are considering a completely rigid vortex chain. If we neglect the oscillations, and just look at the maxima of $U_{\text {pin }}$, it turns out that the pinning strength behaves as $1 /\left|n-n_{0}\right|$ close to commensurate densities $n_{0}$.

The activation energy for the resistance can be estimated as the sum of the amplitude of $U_{\text {pin }}(\bar{x})$, Eq. (26), and the boundary pinning term $E_{b}(n L)$; see Eq. (12). The result is shown in Fig. 4.

Although each vortex is a quantum particle (as we have stressed at the beginning of this section), the possibility of vortex permutations may be safely neglected: for each vortex, $\left\langle\left(x_{i}-x_{i}^{0}\right)^{2}\right\rangle \ll n^{-2}$, i.e., the oscillation amplitude is much less than the intervortex distance.

\section{COMPRESSIBLE VORTEX CHAIN}

In the last section we considered the case of the infinitely long-range vortex-vortex interaction with the consequence that the vortex chain was completely rigid. We will now discuss the importance of screening and the resulting compressibility of the chain.

Screening of the vortex-vortex interaction in the Josephson array is due to two effects: (1) screening by the magnetic field created by currents flowing around the Josephson vortices (Meissner effect), and (2) interaction with the vortex 
$E_{R}$

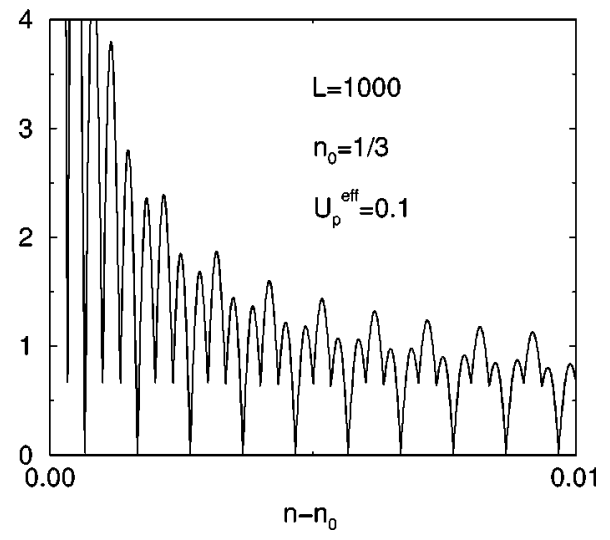

FIG. 4. Activation energy entering the resistance as a function of $n=B W / \Phi_{0}$. The energy plotted here is the sum of the boundary pinning term Eq. (12), and twice the absolute value of the bulk pinning term Eq. (26). Energy units as in Fig. 3, and $n, n_{0}$ are measured in units of $1 / a$.

medium in the contact pads. To start with, we consider the first of these two effects.

The distribution of currents flowing around a vortex depends on the dimensionality of the system. The currents around a vortex line in a three-dimensional superconductor drop off exponentially fast, the characteristic length being the London penetration depth $\lambda_{L}$. For a vortex in a superconducting film of small thickness $s \ll \lambda_{L}$, the screening length $^{17}$ is thickness dependent, $\lambda=\lambda_{L}^{2} / s$. At a sufficiently large distance from the center of a single vortex, $r \gtrsim \lambda$, the spatial distribution of these currents is controlled by the Meissner effect,

$$
j_{1}(r)=\frac{\Phi_{0} c}{4 \pi^{2} r^{2}}
$$

Note, that the distribution (29) is insensitive to the shortscale structure of the two-dimensional vortex: it may be a Josephson vortex in the array, as well as an Abrikosov vortex in the contacts. In both cases, the currents induced by a vortex fall off as $1 / r^{2}$ at sufficiently large distances $r$ from its center, resulting in a vortex-vortex interaction with a finite range $\lambda_{s}$. In the specific case of a quasi-one-dimensional Josephson junction array contacted by superconducting films, we may estimate $\lambda_{s}$ by matching the current density $j_{1}$ with the density $j_{2}$ of the current flowing around a vortex in the array,

$$
j_{2}=\frac{2 \pi^{2} E_{J} c}{\Phi_{0} W} .
$$

The current density $j_{2}$ corresponds to a single-vortex contribution to the phase gradient (5). Equating $j_{1}\left(\lambda_{s}\right) \approx j_{2}$ leads us to the estimate

$$
\frac{\lambda_{s}}{W} \approx \sqrt{\frac{\Phi_{0}^{2}}{8 \pi^{4} E_{J} W}} .
$$

At distances $r \gtrsim \lambda_{s}$, the anisotropy of the system is not important for the current distribution, and Eq. (29) is applicable. At smaller distances, the current distribution is highly anisotropic. In the $x$ direction, the screening length is $\lambda_{s}$, while in the $y$ direction the currents are confined to the thinfilm penetration depth $\lambda$, which depends on the properties of the leads. Typically the sheet superfluid density $\rho_{s}$ in the contacts exceeds greatly the effective superfluid density $\rho_{J}$ in the Josephson junction array, which leads to $\lambda \ll \lambda_{s}$.

The electrodynamic effect limiting the radius of interaction considered above exists for any geometry of the contacts. The estimate (31) does not depend on the characteristics of the contact material. However, the numerical coefficient in Eq. (31) is geometry dependent. In Sec. VI we will further refine the estimate (31) for the specific geometry of the experiment. ${ }^{9}$

In the above consideration, we have completely ignored the existence of Abrikosov vortices in the contacts. This is acceptable only if their currents do not overlap with the currents created by Josephson vortices. The two current density fields are spatially separated if the distance $d$ of the last row of the Abrikosov lattice to the edge of the junction array (see Fig. 1) exceeds $\lambda$. In the opposite case $d \lesssim \lambda$, the Abrikosov lattice effectively truncates the currents created in the contact by a Josephson vortex. Indeed, a small shift of the lattice in the direction perpendicular to the edge of the array is sufficient to compensate these weak currents. To estimate the interaction potential range $\lambda_{s}$ in this case, it is sufficient to deal with the energy of the supercurrents,

$$
E \simeq \frac{4 \pi^{2} E_{J} \lambda_{s}}{W}+\frac{\Phi_{0}^{2} d}{16 \lambda_{s} \lambda},
$$

and neglect the magnetic fields the supercurrents create. In Eq. (32), the first term corresponds to the energy of currents in the array that flow in the region $|x| \lesssim \lambda_{s}$ around the vortex. The second term is the energy of the supercurrents in the contacts. These currents are truncated at the position $d$ $\simeq \sqrt{\Phi_{0} / B}$ of the first row of the Abrikosov lattice, which numerically turns out ${ }^{18}$ to be a sound approximation.

Minimization of the energy equation (32) with regard to $\lambda_{s}$ yields

$$
\frac{\lambda_{s}}{W} \sim \sqrt{\frac{\Phi_{0}^{2}}{32 \pi^{2} E_{J} W} \frac{d}{\lambda}} .
$$

This expression is valid for $d \lesssim \lambda$, and at $d \sim \lambda$ it reasonably well matches the estimate (31). For typical experimental values, Eqs. (31) and (33) yield a screening length for which $n^{-1} \ll \lambda_{s} \lesssim L$. In other words, the vortex-vortex interaction has long, but finite range, and the vortex chain is not completely rigid.

We will now develop a continuum description of the compressible vortex chain, i.e., we will express the energy of the chain in terms of the deviations $u\left(x_{i}^{0}\right)=x_{i}-x_{i}^{0}$, and then go over to a deformation field $u\left(x_{i}^{0}\right) \rightarrow u(x)$. The energy of the chain will be the sum of a bulk pinning term and a boundary pinning term as before. In addition to that, there will be an elastic energy term.

It is straightforward to express the bulk pinning term in terms of $u(x)$ : 


$$
\begin{aligned}
U_{\mathrm{pin}} & =-U_{p}^{\mathrm{eff}} \sum_{i=1}^{N} \cos \left(2 \pi x_{i} / a\right) \\
& \approx-n U_{p}^{\mathrm{eff}} \int_{-L / 2}^{L / 2} d x \cos \left(\frac{2 \pi}{a}(u+\alpha x)\right),
\end{aligned}
$$

where

$$
\alpha=n a\left[\frac{1}{n a}-\mathcal{I}\left(\frac{1}{n a}\right)\right]=\frac{n_{0}-n}{n_{0}}
$$

is a dimensionless measure of the deviation from the commensurate value $1 / n_{0} a=\mathcal{I}(1 / n a)$.

The ends of a long array $\left(L \gg \lambda_{s}\right)$ act on the compressible vortex chain as two independent sources of boundary pinning. In the case of an almost rigid chain, $\lambda_{s} \gg 1 / n$, we can find the pinning potential created by a single end (say, the one corresponding to $x=L / 2$ ) by a slight modification of Eq. (6). Namely, we introduce an exponential factor $\exp [\gamma(x$ $-L / 2)$ ] into the integrand, and replace the lower limit of integration by $-\infty$. After that, we find the extremal value of $\varphi_{0}$ as a function of the position of, say, the last vortex in the chain $x_{N}$, and take the limit $\gamma \rightarrow+0$. This procedure yields

$$
U_{b}(\tilde{u})=\frac{\pi^{2}}{6} \frac{E_{J}}{n W}\left[4(n \tilde{u})^{3}-n \tilde{u}\right] .
$$

Here for convenience we have introduced a new variable $\tilde{u}$ instead of the coordinate $x_{N}$,

$$
\tilde{u} \equiv x_{N}-\left(\frac{L}{2}-\frac{1}{2 n}\right),
$$

and the coordinate $x_{N}$ is no more than one half period away from the end of the array, $|\tilde{u}| \leqslant 1 / 2 n$. The top of the boundary barrier is at $n \tilde{u}=-1 / \sqrt{12}$, and its amplitude is approximately $0.64 E_{J} / n W$.

The elastic energy of vortices, which interact by longrange forces, in the long-wavelength limit takes the form

$$
U_{\mathrm{el}}=\frac{K}{2} \int_{-L / 2}^{L / 2} d x\left(\frac{\partial u}{\partial x}\right)^{2} .
$$

The elastic constant $K$ can be expressed through the vortexvortex interaction potential as

$$
K=n^{2} \int_{-\infty}^{\infty} d x U_{v-v}(x)
$$

Note, that the potential $U_{v-v}(x)$ here is defined differently from Eq. (4). Unlike in Eq. (4), we remove the uncertainty in the definition of the potential by requiring $U_{v-v}(x \rightarrow \pm \infty)$ $=0$.

In order to estimate $K$, we adopt the following model for the interaction potential:

$$
U_{v-v}(x)=\frac{2 \pi^{2} E_{J}}{W} \lambda_{s} \exp \left(-\frac{|x|}{\lambda_{s}}\right),
$$

which correctly reproduces the cusp [cf. Eq. (4)] at $|x|$ $\ll \lambda_{s}$, and reaches zero at $|x| \rightarrow \infty$. Within this model, we find

$$
K \approx n^{2} \int_{-\infty}^{\infty} d x U_{v-v}(x)=\frac{4 \pi^{2} E_{J}}{W}\left(n \lambda_{s}\right)^{2} .
$$

(The real interaction potential in the planar geometry of the contacts considered above falls off as $x^{-2}$, rather than exponentially. However, this should not significantly alter the estimate.) We complete the estimate of $K$ by adopting Eq. (31) for the value of $\lambda_{s}$, which yields

$$
K \approx \frac{\Phi_{0}^{2} n^{2}}{2 \pi^{2}}
$$

The elastic constant becomes softer if the period of the Abrikosov lattice in the contacts is smaller than $\lambda$; see Eq. (33).

Varying $E=U_{\mathrm{el}}+U_{\text {pin }}$ with respect to $u(x)$ leads to the static sine-Gordon equation

$$
K\left(\frac{\partial^{2} u}{\partial x^{2}}\right)-n U_{p}^{\mathrm{eff}} \sin \left(\frac{2 \pi u}{a}-\alpha x\right)=0 .
$$

This equation has been studied in many contexts, e.g., commensurate-discommensurate transitions in adsorbate layers, ${ }^{19}$ here $\alpha$ is the difference of the lattice constants of the substrate and the adsorbate. Another example is the theory of long Josephson junctions ${ }^{20}$ where $\alpha$ is proportional to the magnetic field threading the junction.

\section{PHASES}

The behavior of a one-dimensional vortex chain is closely related to that of an adsorbate layer: ${ }^{19}$ if the magnetic field is commensurate, $n=n_{0}$, the vortex chain is commensurate with the junction array. The activation energy of an elementary excitation at $n=n_{0}$ is given by the energy to push one soliton into the system. The length of such a soliton is given by

$$
x_{s}=\frac{a}{2 \pi} \sqrt{\frac{K}{n U_{p}^{\mathrm{eff}}}},
$$

and its energy is

$$
E_{s}=\frac{4 a}{\pi} \sqrt{K n U_{p}^{\mathrm{eff}}}
$$

A comparison of $x_{s}$ with the interaction radius, Eq. (31), yields $x_{s} / \lambda_{s} \simeq(a / W) \sqrt{n W} \sqrt{0.4 \pi^{2} E_{J} / U_{p}^{\text {eff }}}$; here we have used the estimate (42) for the elastic constant $K$. The applicability of Eq. (43) requires $x_{s} \gtrsim \lambda_{s}$, and therefore Eqs. (44) and (45) are valid only if the pinning potential is reduced by quantum fluctuations compared to its classical value.

On moving $n$ away from $n_{0}$, the magnetic field tries to enforce a period of the vortex lattice that is different from the period of the pinning potential. The chain stays locked to a commensurate state up to a critical value of $\left|n-n_{0}\right|$, or, in other terms, until $|\alpha|$ is less than some critical value $\alpha_{C}$. Below the threshold, at $\left|n-n_{0}\right| \leqslant n_{0}\left|\alpha_{C}\right|$, the activation energy will diminish linearly with increasing $|\alpha|$. This can be seen immediately from the analogous situation in a long homogeneous Josephson junction where quantized fluxons play the role of solitons. The energy to create the first fluxon in the junction has some value at $H=0$, and decreases linearly 
like $-M|H|$ with $|H|$; here $M>0$ is the magnetization of a single fluxon, i.e., a constant. In a complete analogy with this, in our case the energy to create a soliton in the commensurate phase is of the form

$$
E_{s}(n)=E_{s}\left[1-\frac{\left|n-n_{0}\right|}{n_{0} \alpha_{C}}\right] .
$$

Note that the energy $E_{s}(n)$ has a cusplike dependence on $n$ $-n_{0}$. The point $n=n_{0}$ is special: the creation of a soliton or antisoliton costs the same energy. This situation is similar to the line of particle-hole symmetry for a Mott insulator. Deviation from the symmetry point makes creation of solitons or antisolitons preferable. This violation of the symmetry is the origin of the nonanalytic dependence of $E_{s}$ on $n$. The critical values of $\alpha$ at which the soliton energy turns zero are given by

$$
|\alpha|=\alpha_{C} \equiv \frac{4}{\pi} \sqrt{\frac{n U_{p}^{\mathrm{eff}}}{K}}=\frac{8 a}{x_{s}} .
$$

Above the threshold, at $|\alpha|>\alpha_{C}$, discommensurations will exist: the chain is strained in the discommensurations, but this is offset by the fact that the rest of the chain can stay in the minima of the pinning potential. In this incommensurate phase, the concentration of solitons is finite. Due to the solitons, the vortex chain regains a finite compressibility $K_{s}$, which depends on how far the system is tuned away from the critical points $\alpha= \pm \alpha_{C}$. Without giving the details here, we note that the dependence of the renormalized elastic constant on the control parameter $\alpha$ can be presented in a parametric form, ${ }^{20}$ as follows:

$$
\begin{gathered}
\frac{K_{s}}{K}=\frac{4}{\pi^{2}} \frac{\frac{d}{d \gamma}[E(\gamma) / \gamma]}{\frac{d}{d \gamma}[1 / \gamma K(\gamma)]}, \\
\frac{|\alpha|}{\alpha_{C}}=\frac{E(\gamma)}{\gamma} .
\end{gathered}
$$

Here $K(\gamma)$ and $E(\gamma)$ are the complete elliptic integrals of the first and second kind, respectively. The chain softens near the critical points, where the proper expansion ${ }^{19}$ of Eq. (48) yields

$$
\frac{K_{s}}{K}=\frac{8}{\pi^{2}} \frac{|\alpha|-\alpha_{C}}{\alpha_{C}}\left[\ln \frac{\alpha_{C}}{|\alpha|-\alpha_{C}}\right]^{2} .
$$

The softening occurs, because the solitons in the chain are rare, and the pair potential acting between them is exponentially small, $U_{s} \sim E_{s} \exp \left(-x / x_{s}\right)$. Far away from the transition, at $\left|n-n_{0}\right| \gg \alpha_{C} n_{0}$, the solitons overlap, and $K_{s}=K$.

A finite voltage between the contacts to the array (see Fig. 1) is related, by the Josephson relation, to the average velocity of vortices moving along the array. The transport of a vortex through the system can be viewed as propagation of solitons through the vortex chain. The availability of solitons in the chain will clearly affect the resistance of the array. In the commensurate phase, the soliton density is exponentially small at low temperatures, $E_{s}(n)$ being the corresponding activation energy. In the incommensurate phase, there are mobile solitons in the system even at zero temperature. If one neglects the existence of boundary effects, the activation energy $E_{R}$ of the observable quantity, viz., resistance, would coincide with the activation energy of a single soliton. Hence, one would expect $E_{R}=E_{s}(n)$ in the commensurate phase, and $E_{R}=0$ in the incommensurate phase.

It turns out, however, that boundary pinning modifies this picture. First of all, it may affect the ground state of the vortex chain. In the incommensurate phase, even weak boundary pinning will lead to a deformation of a long compressible chain. In the commensurate phase, the structure of the ground state starts to depend on the ratio of the boundary pinning energy $E_{b} \sim E_{J} / n W$, and the soliton energy $E_{s}(n)$; this ratio depends on the bare parameters of the system, and may be small or large. Second, the set of excited states the chain goes through during the elementary act of a vortex transfer also depends on the boundary pinning. These two factors determine the dependence of $E_{R}$ on the characteristic energies $E_{s}$ and $E_{b}$. We will analyze the activation energy $E_{R}$ for both cases of small and large value of this ratio.

In the commensurate phase, and in the presence of strong boundary pinning, $E_{b}>E_{s}(n) / 2 n a$, the vortex chain in the ground state will adjust itself to the length of the array to minimize the pinning energy. This means there are solitons in the ground state, unless $n L$ is an integer. The largest number of solitons in the ground state occurs at a half-integer value of $n L$, and equals $1 / 2 n a$. In this particular case, the chain without solitons is the configuration with the highest energy that the system passes through during a vortex transfer. In this state, the chain is not adjusted to the boundary of the array, and the boundary pinning energy attains its maximum value $E_{b}$. The difference of this energy from the ground state is $E_{R}=E_{b}-E_{s}(n) / 2 n a$. In the opposite case of integer $n L$, there are $1 / 2 n a$ solitons in the "saddle-point" state, and the activation energy reaches its maximum, $E_{R}=E_{b}+E_{s}(n) / 2 n a$; see Fig. 5(a).

If $E_{b}<E_{s}(n)$, there are no solitons in the ground state of a commensurate chain. Moreover, during the process of a vortex transfer through the array, there is at most one soliton in the chain. Since a soliton changes the length of the vortex chain only by $a$, the chain remains rigid on the scale of the intervortex distance $1 / n$. Therefore, we arrive at the following picture of the vortex transfer. The passage of each soliton shifts the chain by $a$. The transfer of a vortex requires the sequential passing of $1 / n a$ solitons. In this process, the chain moves as a rigid object in the presence of boundary pinning. Thus, $E_{R}$ is the sum of $E_{s}(n)$ and the boundary pinning energy equation (12) for a rigid chain,

$$
E_{R}=E_{s}(n)+E_{b}(n L)
$$

see Fig. 5(b). The soliton energy (46) vanishes at the boundaries of the commensurate phase. Before it vanishes, we cross over to the case described in the previous paragraph.

We will now discuss the incommensurate phase, $n>n_{C}$. At the phase transition, the soliton formation energy vanishes, and solitons will start to form spontaneously. Correspondingly, the physics of the incommensurate phase will be determined by boundary pinning and by the elastic energy, and the behavior of the activation energy will be identical for 

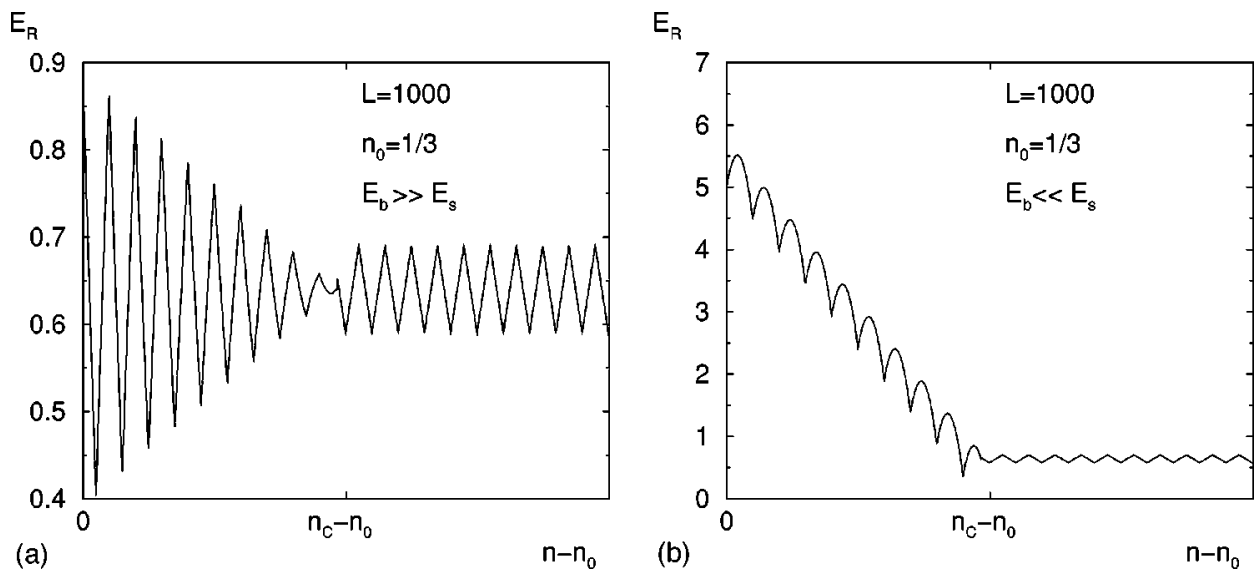

FIG. 5. Phase diagram of the compressible vortex chain: activation energy as a function of $n-n_{C}$. (a) $E_{b} \gg E_{s}$, i.e., boundary pinning dominates $\left(E_{s}=0.5\right)$. (b) $E_{b} \ll E_{s}$, i.e., soliton formation energy dominates $\left(E_{s}=5\right)$. Energy units as in Fig. 3, and the one-dimensional vortex densities $n, n_{0}, n_{C}$ are measured in units of 1/a. On the incommensurate side of the transition, $n>n_{C}$, solitons will form spontaneously. The physics of the incommensurate phase is therefore determined by boundary pinning and the elastic energy and is the same for (a) and (b).

the two panels of Fig. 5. The chain is compressible; the elastic constant $K_{s}(n)$ is renormalized down by solitons; see Eq. (48). The adjustment of the vortex chain to the length of the array leads to a finite deformation. The corresponding elastic energy can be found with the help of Eq. (38) with $K$ replaced by $K_{s}(n)$. The maximum value of the deformation $\partial u / \partial x=1 / 2 n L$ corresponds to half integer $n L$, and the elastic energy associated with it is $K_{s}(n) / 8 n^{2} L$. For large $L$, this energy is inevitably smaller than the boundary pinning potential (36). To initiate an elementary act of vortex transport through the array, a shift of the end vortex through the maximum of the potential (36) should occur. This varies the deformation of the chain by $1 / n \sqrt{3}$, or by $(1-1 / \sqrt{3}) / n$. The corresponding elastic energy in both cases is the same, and is equal to $K_{s}(n)(1-2 / \sqrt{3})^{2} / 8 n^{2} L$. The net variation of the elastic energy involved in the described shift of the vortex is

$$
\delta U_{\mathrm{el}}=\frac{K_{s}(n)}{8 n^{2} L}\left[\left(1-\frac{2}{\sqrt{3}}\right)^{2}-1\right] \approx-0.12 \frac{K_{s}(n)}{n^{2} L} .
$$

To obtain the activation energy for the resistance $E_{R}$ at this particular value of $n L$, one should add $\delta U_{\mathrm{el}}$ to the boundary pinning amplitude. At some other values of $n L$, the variation in the elastic energy involved in the process of passing the boundary barrier attains its maximum value $-\delta U_{\mathrm{el}}$. Thus, the resistance activation energy oscillates between two values,

$$
E_{R} \approx 0.64 \frac{E_{J}}{n W} \pm 0.12 \frac{K_{s}(n)}{n^{2} L},
$$

with the period $\Delta n=1 / L$.

\section{COMPARISON WITH THE EXPERIMENT}

The resistance $R$ of a number of arrays of Josephson junctions was measured in the geometry depicted in Fig. 1 in the presence of a magnetic field. Arrays with lengths $L$ varying between $100 a$ and $1000 a$, and widths $W$ of $7 a$ and $3 a$ were studied. The characteristic Josephson energy for all the samples was about $1 \mathrm{~K}$, with the ratio $E_{J} / E_{C}$ varying within the limits 0.7 to 2.8 . (The details of sample preparation as well as the experimental techniques can be found in Ref. 9). The main qualitative feature of the field dependence of $R$ consists in the existence of a finite region of magnetic flux densities $n$ around the commensurate value $n_{0}=1 / 3 a$, where the resistance is strongly suppressed (Mott phase for the system of quantum vortices). The width of this region becomes smaller with the increase of the "quantum parameter", $E_{C} / E_{J}$, see Ref. 9, in agreement with the notion of the Mott transition.

Within the Mott phase, the resistance clearly displays an activated behavior, with the activation energy $E_{R}$ strongly depending on the deviation $\left|n-n_{0}\right|$ from the point of exact commensurability. In Fig. 6 we present new data for the activation energy for our longest sample, $L=1000 a$, with parameters $W=7 a, E_{C}=0.7 \mathrm{~K}$, and $E_{J}=0.9 \mathrm{~K}$. For each value of $n$, the activation energy $E_{R}$ was determined from the measured temperature dependence of the array resistance. The measurement was performed in the linear regime, at a small transport current. For this sample, the commensurate phase around the point $n_{0}=1 / 3 a$ exists in the domain $|\alpha|$ $<\alpha_{C} \approx 0.009$. The maximal value of the activation energy, $E_{R} \approx 12 \mathrm{~K}$, is reached at the commensurability point. Outside the Mott phase region, the resistance exhibits strong oscillations; the activation energy vanishes almost periodically with the period $\Delta n=1 / L$. We find two aspects of this data striking.

First, the regions of $n$ corresponding to the Mott phase are extremely narrow $\left(\alpha_{C} \simeq 10^{-2}\right.$ ). In the conventional picture, this would imply a weak interaction between the particles (compared to the one-particle band-structure energies). Consequently, within the Mott phase the activation energies for particle transport must be also small. Quite contrary, the observed value of the resistance activation energy is about one order of magnitude larger than the energies $E_{C}$ and $E_{J}$, which determine the single-vortex band spectrum.

Second, the resistance $R(n)$ exhibits strong oscillations with the period $\Delta n=1 / L$ outside the Mott region. These oscillations would not be expected in a model of almost-free 


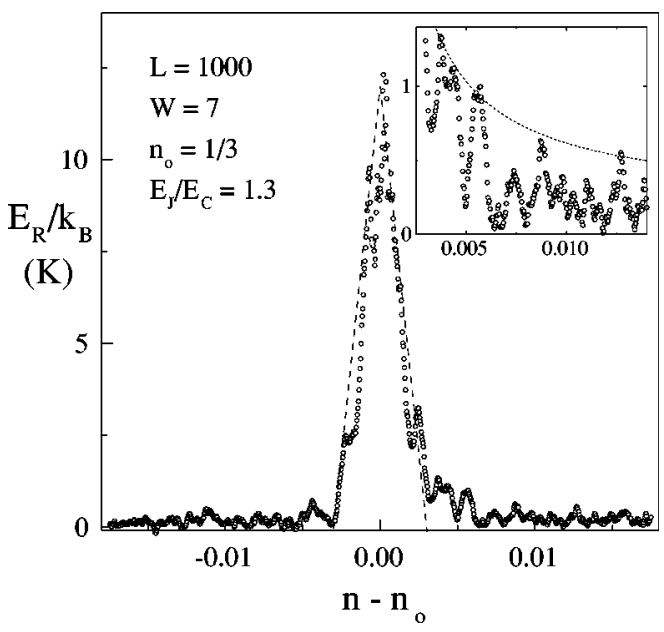

FIG. 6. Activation energy of the resistance of an array consisting of $1000 \times 7$ cells with parameters $E_{J}=0.9 \mathrm{~K}$ and $E_{C}=0.7 \mathrm{~K}$. The one-dimensional vortex densities $n, n_{0}$ are measured in units of $1 / a$. The dashed line is a fit to the data to extract the width of the Mott region. The cusplike part of the figure corresponds to the Mott phase. Additional wiggles on that part may be related to boundary effects; see Sec. V and Fig. 5(b). Inset: activation energy outside the Mott phase. The zeros of $E_{R}(n)$ at $n-n_{0}>0.006$ indicate the restored rigidity of the vortex chain. Note that in qualitative agreement with Fig. 4 the maxima of $E_{R}(n)$ decrease with increasing $\left|n-n_{0}\right| ;$ the dotted line in the inset is a guide to the eye.

quasiparticles within the delocalized phase.

These two observations find a natural explanation in our model, which explicitly accounts for the long-range interaction forces between the vortices.

In order to perform a detailed quantitative comparison of the theory and experiment, first we improve the estimates (31) and (42) for the interaction range $\lambda_{s}$ and the elastic constant $K$, respectively. In the experiment, ${ }^{9}$ the contact bars were made by shorting the junctions at the lower and upper border of the array. We therefore model the bars by superconducting strips of average width $a \approx 10^{-4} \mathrm{~cm}$ (the lattice constant of the array). The condition $\lambda_{s} \gg W$ allows us to neglect nonlocal effects in the solution of the magnetostatic problem, ${ }^{21}$ and to express $\lambda_{s}$ in terms of the selfinductance $\mathcal{L}$ of the two-wire system. In addition, as the distance $W$ between the wires exceeds significantly their width $a$, we can use the textbook ${ }^{22}$ formula $\mathcal{L}=4 \ln (W / a)$. As a result, Eq. (31) is replaced by

$$
\lambda_{s}=W \sqrt{\frac{\Phi_{0}^{2}}{16 \pi^{2} E_{J} W \ln (W / a)}} \simeq 270 a .
$$

(The resulting numerical value here refers to the parameters of the sample of Fig. 6.) In the same approximation, the model form of the potential (40) becomes exact. With the help of Eq. (53), the estimate (42) of the elastic constant can also be refined. For convenience, we give here the value of the product $a K$, instead of the value of the elastic constant:

$$
a K \approx \frac{\Phi_{0}^{2} n^{2} a}{4 \ln (W / a)} \simeq 3.5 \times 10^{4} \mathrm{~K} .
$$

The theoretical results (53) and (54) do not have any adjustable parameters, and are obtained within controllable ap- proximations. In contrast with this, Eq. (24) for the effective pinning cannot be used for the quantitative comparison with the experiment: ${ }^{9}$ The tight-binding approximation we have used in Section III to estimate the suppression of the pinning potential is not applicable to the case $E_{C} \approx E_{J}$. [We note that Eq. (23) still allows one to reproduce the correct trend in the variation of $\alpha_{C}$ with the ratio $E_{C} / E_{J}$.] Therefore, we proceed in the following way. First, we find the soliton length $x_{s}$ from the experimental values of $\alpha_{C}$. Then, using the theoretical value of $a K$ and the value of $x_{s}$ extracted from the data, we find the renormalized pinning potential $U_{p}^{\text {eff }}$ and the soliton activation energy $E_{s}$. We will check that the renormalized pinning potential is indeed substantially lower than its bare value $0.1 E_{J}$. Finally, we will relate the found value of $E_{s}$ to the activation energy of the resistance for the experimental sample.

The values of $\alpha_{C}$ and $a \approx 10^{-4} \mathrm{~cm}$ found experimentally allow us to estimate the soliton length from Eq. (47):

$$
x_{s}=\frac{8 a}{\alpha_{C}}=890 a \approx 0.09 \mathrm{~cm} .
$$

This length is really large. In fact, $x_{s}$ is about three times the length of the array $L=300 a$ used in Ref. 9 to extract the activation energy $E_{R}$. This may explain why the values of $E_{R}$ found there are systematically lower than the activation energy for the longest array; see Fig. 6. A single soliton consists of about 300 vortices, and therefore its activation energy may exceed easily the single-vortex energy scales. Note also that $x_{s}$ exceeds considerably the interaction radius (53) that gives us confidence in the applicability of the sineGordon equation (43). The effective pinning potential, according to Eq. (44), can be found as

$$
U_{p}^{\mathrm{eff}}=\left(\frac{a}{2 \pi x_{s}}\right)^{2} \frac{a K}{n a} \simeq 3.4 \times 10^{-3} \mathrm{~K} .
$$

This energy is at least one order of magnitude smaller than its bare value $0.1 E_{J}$; see Eq. (3). The reduction is apparently due to the quantum zero-point motion of individual vortices. Again, the strong suppression of the pinning energy guarantees the harmonic form of the pinning potential (23), and hence allows us to use the sine-Gordon equation for the solitons. Finally, using Eqs. (44) and (45), we find the soliton energy at the commensurability point:

$$
E_{s}=\frac{\alpha_{C}}{4 \pi^{2}} a K \simeq 8 \mathrm{~K}
$$

This energy exceeds significantly the boundary pinning energy. According to Eqs. (12) and (50), the latter contributes to $E_{R}$ less than $0.5 \mathrm{~K}$. We neglect this contribution, and therefore identify $E_{R}$ with the energy $E_{s}$ of the formation of a soliton. The calculated value (57) is somewhat lower than the measured $E_{R}$. Still, we find the agreement quite impressive, having in mind the huge value of the elastic constant (54), calculated without any adjustable parameters.

The large value of the elastic constant (54) results from the long-range nature of the intervortex interaction forces. In fact, the vortex chain in the incommensurate phase is so rigid that Eq. (52) is inapplicable in the case of a sample only a thousand cells long. Away from the transition point 
$\left(|\alpha|=\alpha_{C}\right)$, the elastic term in Eq. (52), which is supposed to be a small correction, is about 70 times larger than the "main" boundary pinning term. Therefore, for the conditions of the experiment, the incommensurate phase is well described by the model of a rigid vortex chain; see Sec. II. This immediately explains the strong oscillations of the activation energy with the period $\Delta n=1 / L$ in the incommensurate phase, see Eqs. (12) and (26).

There is a clear resemblance between the experimentally measured curve of $E_{R}(n)$ (inset in Fig. 6), and the curve in Fig. 4, simulated with the help of Eqs. (12) and (26). In agreement with the model of a rigid chain, the minima of $E_{R}(n)$ reach zero at $\left|n-n_{0}\right| \geqslant 0.005$, and the maxima of $E_{R}(n)$ decrease with increasing $\left|n-n_{0}\right|$. The boundary term Eq. (12) has a maximum of $\approx 0.5 \mathrm{~K}$ and accounts for the main contribution to the maxima of $E_{R}$ at $n-n_{0}>0.006$. The vortex chain softens up only in a very narrow region around the transition point, so that the crossover region is of the order of $\Delta n$; see the inset in Fig. 6.

To end this section, we reiterate that in the experiment the commensurability point $n_{0}=1 / 3 a$ was reached in the sample with $W=7 a$, which means the chain is stable against the formation of a zigzag structure at $1 / n W=0.43$. According to Eq. (15), for a continuous system, the zigzag instability would already occur at $1 / n W=0.65$, i.e., before the density $n_{0}=1 / 3 a$ is reached. Since the experimental data show no indication of a qualitative difference between the arrays with $W=3 a$ and $W=7 a$, we conclude that the array width $W$ $=7 a$ is narrow enough to allow suppression of the instability by the effects of discreteness.

\section{DISCUSSION}

An external magnetic field applied to an array of Josephson junctions allows one to introduce vortices into it. A sufficiently weak field creates a linear chain of vortices in the quasi-one-dimensional array. The ratio between the periods of the vortex chain and the array of Josephson junctions is controlled by the value of the magnetic field. The commensurate phase corresponds to the vortex analog of a Mott insulator. Within this phase, the elementary excitation is a soliton consisting of a number of individual vortices. The finitegap energy for the soliton translates into a finite activation energy of the resistance of the array. Each soliton transfers a fraction of the flux quantum through the array. In the incommensurate phase, the spontaneous proliferation of solitons and antisolitons leads to the formation of a one-dimensional vortex liquid. This results in a finite vortex-flow resistance of the array.

In this paper we have analyzed the commensurateincommensurate transition for a one-dimensional vortex system in detail. The size and energy of the vortex solitons, which drive the transition, depend on two parameters of the vortex chain. These parameters are the elastic constant and the pinning potential existing due to the discreteness of the array of Josephson junctions. The long-range nature of the vortex-vortex interaction leads to a large value of the elastic constant. On the other hand, the zero-point motion of each quantum vortex leads to a considerable suppression of the pinning potential. As a result, the size of the solitons turns out to be extremely large, about 300 vortices under the conditions of the experiments reported in this paper and in Ref. 9. This enables us to treat the transition in the framework of the classical theory. ${ }^{19}$ Our theory explains quantitatively the main experimental observations.

We would like to conclude with the following remark: a quasi-one-dimensional array of small superconducting islands connected by Josephson junctions can be used to study quantum phase transitions in two complementary ways. The first way relies on the control of the charge state of the islands by an external gate. In this case, a transition between the charge-localized and charge-delocalized phases can be observed in principle. The localized phase is a Mott insulator, with a finite gap for charge solitons, which play the role of elementary excitations. The delocalized phase behaves as a one-dimensional Luttinger liquid (see, e.g., Ref. 23 and references therein). The experimental observation of the two phases and the transition is difficult, as it is virtually impossible to avoid the existence of random offset charges, which introduce strong disorder into the system. The other way is to study the commensurate-incommensurate transition in a system of vortices induced in the array by an external magnetic field (the case studied in this paper). This transition belongs to the same universality class as the Mott transition for charge delocalization. A great advantage of the vortex system is that it is virtually disorder free. However, due to the large size of the solitons driving the transition, the critical region around the phase transition point is extremely narrow for the arrays studied experimentally. To widen the critical region, one should find a way to reduce the vortex-vortex interaction strength. That would open new possibilities of experimental investigations of the Luttinger liquid that is formed on the incommensurate side of the transition. The properties of the liquid are expected to depend crucially ${ }^{24}$ on the value of the fractional flux carried by the solitons.

\section{ACKNOWLEDGMENTS}

We would like to thank R. Fazio, K. K. Likharev, Yu. Makhlin, Yu. V. Nazarov, and T. Orlando for discussions. C.B. would like to thank the Theoretical Physics Institute of the University of Minnesota for its hospitality. L.G. acknowledges hospitality of Delft Technical University where part of this work was performed. The work at the University of Minnesota was supported by NSF Grants No. DMR9731756 and No. DMR-9812340.
*Present address: Department of Physics and Astronomy, University of Basel, Klingelbergstrasse 82, CH-4056 Basel, Switzerland.

${ }^{1}$ E. Simanek, Inhomogeneous Superconductors (Oxford University Press, New York, 1994).

${ }^{2}$ R. Fazio and G. Schön, Phys. Rev. B 43, 5307 (1991).

${ }^{3}$ L. J. Geerligs, M. Peters, L. E. M. de Groot, A. Verbruggen, and J E. Mooij, Phys. Rev. Lett. 63, 326 (1989).

${ }^{4}$ A. I. Larkin, Yu. N. Ovchinnikov, and A. Schmid, Physica B 152, 266 (1988).

${ }^{5}$ U. Eckern and A. Schmid, Phys. Rev. B 39, 6441 (1989).

${ }^{6}$ H. S .J. van der Zant, F. C. Fritschy, W. J. Elion, L. J. Geerligs, and J. E. Mooij, Phys. Rev. Lett. 69, 2971 (1992). 
${ }^{7}$ For a number of articles, see Proceedings of the NATO Advanced Research Workshop on Coherence in Superconducting Networks, edited by J. E. Mooij and G. Schoön [Physica B 152, 1 (1988)].

${ }^{8}$ C. J. Lobb, D. W. Abraham, and M. Tinkham, Phys. Rev. B 27, 150 (1983).

${ }^{9}$ A. van Oudenaarden and J. E. Mooij, Phys. Rev. Lett. 76, 4947 (1996); A. van Oudenaarden, B. van Leeuwen, M. P. M. Robbens, and J. E. Mooij, Phys. Rev. B 57, 11684 (1998).

${ }^{10}$ M. P. A. Fisher, P. B. Weichman, G. Grinstein, and D. S. Fisher, Phys. Rev. B 40, 546 (1989).

${ }^{11}$ M. Kardar, Phys. Rev. B 33, 3125 (1986).

${ }^{12}$ The conditions under which the superconductor-insulator transition occurs were considered in a number of papers that study a more general model, which includes the capacitance $C_{g}$ of the grains to a gate. Probably the most reliable estimate of the critical value of $E_{C} / E_{J}$ for the limiting case $C_{g} \rightarrow 0$ considered here is provided by direct Monte Carlo simulation; see J. V. Jose and C. Rojas, Physica B 203, 481 (1994). The simulation yields $E_{C} / E_{J} \approx 1.4$.

${ }^{13}$ P. G. De Gennes, Superconductivity of Metals and Alloys (W. A. Benjamin, New York, 1966).

${ }^{14}$ Taking into account the vortex core energies leads to a small field-independent shift in the argument of the function $\mathcal{I}(x)$. We neglect this shift here and in the rest of the paper.

${ }^{15}$ K. K. Likharev, Zh. Eksp. Teor. Fiz. 61, 1700 (1972) [Sov. Phys. JETP 34, 906 (1972)].

${ }^{16}$ L. D. Landau and E. M. Lifshitz, Statistical Physics: Part II (Pergamon Press, Oxford, 1980), p. $230 f$.

${ }^{17}$ J. Pearl, Appl. Phys. Lett. 5, 65 (1964).

${ }^{18}$ F. F. Ternovskii and L. N. Shekhata, Zh. Eksp. Teor. Fiz. 62, 2297 1982) [Sov. Phys. JETP 35, 1202 (1972)].

${ }^{19}$ P. M. Chaikin and T. C. Lubensky, Principles of Condensed Matter Physics (Cambridge University Press, Cambridge, 1995).

${ }^{20}$ I. O. Kulik and I. K. Yanson, The Josephson Effect in Superconducting Tunneling Structures (Israel Program for Scientific Translations, Jerusalem, 1972).

${ }^{21}$ R. D. Bock, J. R. Phillips, H. S. J. van der Zant, and T. P. Orlando, Phys. Rev. B 49, 10009 (1994).

22 J. D. Jackson, Classical Electrodynamics (Wiley, New York, 1975), p. 262.

${ }^{23}$ L. I. Glazman and A. I. Larkin, Phys. Rev. Lett. 79, 3736 (1997).

${ }^{24}$ M. P. A. Fisher and L. I. Glazman, in Mesoscopic Electron Transport, edited by L. L. Sohn, L. P. Kouwenhoven, and G. Schön (Kluwer, The Netherlands, 1997), p. 331. 